\title{
The Cruciform Drain: A Technical Note on the Surgical Management of Cystic Lesions of the Sella
}

Ivan Cabrilo, MD, FEBNS, ${ }^{1}$ Hani J. Marcus, PhD, FRCS, ${ }^{1}$ Neil L. Dorward, FRCS, $\mathrm{MS}^{1}$

${ }^{1}$ Department of Neurosurgery, National Hospital for Neurology and Neurosurgery, Queen Square, London, United Kingdom

\section{Corresponding author: Ivan Cabrilo}

- Affiliation: Department of Neurosurgery, National Hospital for Neurology and Neurosurgery, Queen Square, London, United Kingdom

- E-mail: iv.cabrilo@gmail.com

- Address: Neurosurgery Department, National Hospital for Neurology and Neurosurgery, Queen Square, WC1N 3BG London, United Kingdom

- ORCID: 0000-0002-3875-0355

\section{Funding:}

No specific funding was received for this research. Hani J Marcus is supported by the Wellcome / EPSRC Centre for Interventional and Surgical Sciences (WEISS) and the National Institute of Health Research (NIHR) University College London (UCL) Biomedical Research Centre (BRC).

\section{Disclosure of interest:}

The authors report no conflict of interest. 


\section{The Cruciform Drain: A Technical Note on the Surgical Management of Cystic Lesions of the Sella}

Background: The postoperative recurrence of cystic lesions of the sella is frequent and may require further surgery for re-drainage.

Objective: To tackle this problem, we propose to insert a small cross-shaped drain coursing from the cyst lumen to the suprasellar cistern. At this early stage of innovation, the technique is primarily intended for patients who present with a recurrence.

Methods: The cruciform drain is fashioned from the tip of a ventricular catheter and is inserted under endoscopic vision. We retrospectively reviewed the pre- and postoperative records of patients in whom this technique was implemented.

Results: A cruciform drain was placed in 5 patients since the introduction of the technique into our practice in 2018. The use of the cruciform drain did not impact upon the expected surgical workflow nor was it associated with adverse intraoperative events, but 3 patients did develop a postoperative CSF leak that was successfully treated in all cases. None of the patients showed re-collection of their cysts on early radiological follow-up.

Conclusion: The cruciform drain is intended to prevent the renewed build-up of cystic fluid by allowing it to flow through and around the drain into the subarachnoid space. We have modified our repair protocol in response to the observed high CSF leak rate, as a basis for further development of the technique. Studies involving long-term follow-up will also be required to assess its efficacy in reducing cyst recurrence. 
Keywords: cyst fenestration; permanent drain; prevention of recurrence; sellar cystic lesions; transsphenoidal surgery

Abbreviations:

CSF: $\quad$ Cerebrospinal fluid

ACTH: $\quad$ Adrenocorticotrophic hormone

MRI: $\quad$ Magnetic resonance imaging

RCC: $\quad$ Rathke's cleft cyst

AC: $\quad$ Arachnoid cyst

Word count: 2938. 


\section{Introduction}

The recurrence of cystic lesions of the sella after transsphenoidal drainage is frequent, with rates of up to $40 \%$ in cases of Rathke's cleft cysts (RCC) and sellar arachnoid cysts (AC) having been reported in the literature. ${ }^{1-6}$ Various measures have been proposed to address this problem, including radical excision of the cyst wall ${ }^{7,8}$ or simple fenestration of the cyst towards the subarachnoid space..$^{9-12}$

Radical cyst excision, however, is associated with a high risk of postoperative pituitary dysfunction, ${ }^{4,5,13-17}$ and both radical cyst excision and simple fenestration come at the expense of an increased risk of postoperative cerebrospinal fluid (CSF) leak. ${ }^{3,12}$ Moreover, with regard to cyst fenestration, it seems reasonable to posit that a more modest opening towards the suprasellar cistern may be associated with a lesser risk of postoperative CSF leak. Yet, it is similarly plausible that such an aperture comes at a risk of subsequent closure if it is too small, ${ }^{11}$ thereby depriving the surgery of its intended effect.

With these considerations in mind, here we present our initial use of a small cross-shaped drain in the surgical management of select cystic sellar lesions, with the aim of preventing cyst recurrence by establishing a small but nonetheless long-term stoma between the cyst's lumen and the suprasellar subarachnoid space. The technique described in this report is at Stage 1 of Surgical Innovation (i.e. Idea phase) according to the "Innovation, Development, Exploration, Assessment and Long-Term Study (IDEAL) model" statements. ${ }^{18}$ As such it is intended as a proof of feasibility and a report on initial outcome that will provide the basis for future adaptations and work. 


\section{Methods}

All procedures performed in studies involving human participants were in accordance with the ethical standards of the institutional and/or national research committee and with the 1964 Helsinki declaration and its later amendments or comparable ethical standards.

This retrospective study was registered as a Service Evaluation study with our institution's Clinical Audit Committee.

Where applicable, this report follows the "Strengthening the Reporting of Observational Studies in Epidemiology (STROBE) Statement" checklist ${ }^{19}$ and the "Innovation, Development, Exploration, Assessment and Long-Term Study (IDEAL) model" statements. ${ }^{18}$

\section{Indications}

The technique applies to recurrent cystic lesions with serous contents amenable to drainage (RCC, AC), or eventually other select cases based on the surgeon's assessment of the patient's radiological presentation and intraoperative findings (e.g. Patients 3 and 5, in Table 1). If the cystic lesion is amenable to resection at a low risk of disturbance to the pituitary gland, this approach is preferred, and no surgical adjunct is used.

\section{Description of the Surgical Technique}

All patients are operated through an endoscopic endonasal approach. Once upon the lesion, the cyst is fenestrated largely over its antero-inferior aspect (Fig. 1A-C) and a smaller fenestration is made in its supero-posterior aspect, into the suprasellar subarachnoid space (Fig. 1D). 
We fashion the cruciform drain (Fig. 2) from the tip of a ventricular shunt catheter (Silverline ${ }^{\circledR}$ Spiegelberg; Hamburg, Germany), by making a small vertical incision - in line with the long axis of the drain - through which a second, smaller section of drain is then inserted. In fact, any soft catheter with perforations can in theory be used for this purpose - including an external ventricular drain. The advantage, however, of a shunt ventricular catheter here is that it is a material that is licensed for permanent - rather than temporary - use.

The resulting, cross-shaped drain has holes along its length, and is introduced through both fenestrations so as to straddle the cyst's lumen and the suprasellar cistern (Fig. 3). The drain is individually tailored to the sellar anatomy of a given patient and their cyst (Fig. 2). Its length is customised so that it is not too short to retract into the cyst's lumen and so fall backwards, out of the fenestration; but at the same time not too long, in order to avoid it protruding through the sellar floor and risking it being impacted deeper than intended into the subarachnoid space during sellar reconstruction and graft application. The crosspiece lies transversally against the cyst's wall to prevent the drain from sliding in deeper. But it also acts as ballast to the drain, preventing rotational movement and so preventing the drain from "wriggling" out of place.

For closure, fat, harvested from the abdomen, is placed over the sellar opening in such a way as to plug it, but not as to fill the pituitary fossa. Fibrin glue is applied over this, and the process is repeated with a second piece of fat. An interface of absorbable gelatin sponge is placed on the surface of this graft, and ribbon gauze, doused in bismuth paste, is used for packing of the nostril. A lumbar drain is inserted, to be removed five days after surgery. 


\section{Patients and data}

All patients were included in whom a cruciform drain had been implanted since the introduction of the technique by the senior author (NLD) in 2018. This retrospective cohort study assessed whether the use of the cruciform drain was associated with adverse intraoperative events, and reviewed patients' demographic information and pre- and postoperative clinical and radiological records for diagnosis of sellar pathology, pituitary hormone deficiency, status of vision, postoperative CSF leak and its management, overall performance status at the first postsurgical clinical follow-up (at 3 months), radiological evolution of anterior optic pathway compromise and confirmation of the intended and nondisplaced position of the cruciform drain.

\section{Results}

These are summarised in Table 1.

\section{Preoperative patient description (demographical, clinical and radiological)}

Five patients were included. Three patients presented with visual field defects, and anterior pituitary insufficiency (hypogonadism) was found in 2. Magnetic resonance imaging (MRI) revealed suprasellar extension in all cases, causing compression of the anterior optic apparatus. The diagnosis was RCC in 2 patients, $\mathrm{AC}$ in 2 patients, and non-functioning pituitary macroadenoma with corticotroph expression in the remaining patient. Three lesions were recurrent: One AC had previously been drained on three occasions (17, 10 and 6 years before the surgery reported here), including once transcranially; The AC in the second patient had recurred clinically and radiologically only six months after its initial simple drainage, also through the transsphenoidal route; And one patient presented with a radiological recurrence of a RCC operated nine years earlier. 


\section{Adverse intraoperative events}

Surgery was uneventful in all cases.

\section{Postoperative CSF leak}

Postoperatively, 2 patients presented with a CSF leak three days after the removal of their

lumbar drains, and both were successfully treated with another 5-day course of lumbar drainage. One patient presented with a CSF leak following an outburst of sneezes a month after his procedure and underwent a redo endoscopic transsphenoidal repair with a 5-day course of lumbar drainage, with resolution of the leak.

\section{Postoperative pituitary hormone deficiency}

All but one patient were found to be adrenocorticotrophic hormone (ACTH) deficient on serum cortisol dosage performed on postoperative day 2 (cut-off value of $350 \mathrm{nmol} / \mathrm{L}$ ), and were initiated on hydrocortisone replacement therapy. This was stopped in 1 patient who was subsequently found to have sufficient ACTH reserve on endocrinological follow-up. No patient developed diabetes insipidus.

\section{Postoperative visual status}

Improvement in vision was documented in all but 1 case with visual deficits: In this patient, the preoperative visual field findings (bilateral tunnel vision) were not typical for chiasmatic compression. Nonetheless, in the presence of a radiologically significant compression, surgery had been offered to the patient. 


\section{Postoperative clinical and radiological follow-up}

All patients were well on clinical follow-up at 3 postoperative months with a Karnofsky performance score of 100 for Patients 1,2, 4 and 5 and of 90 for Patient 3 who continued to suffer from bilateral tunnel vision. A follow-up MRI was performed 6 months following surgery (Fig. 4), showing for each case a successful decompression of the optic apparatus, no radiologically identifiable complication and no displacement of the cruciform drain. Followup is at 19 months at its current longest.

\section{Discussion}

\section{Principle findings}

With the frequent recurrence of sellar cystic lesions in mind, we propose to place a small transcysto-cisternal drain through a fenestration towards the subarachnoid space, as an adjunct to the transsphenoidal drainage of these lesions. Representing work at Stage 1 - Idea phase - of Surgical Innovation (IDEAL model),${ }^{18}$ here we demonstrate this technique's feasibility and discuss strategies to address the encountered shortcomings in the future.

\section{Recurrence rates in the literature}

The reported postoperative recurrence rates range from $10 \%$ to $40 \%$ for $\mathrm{RCC}^{1-5,7,13,15,17,20-26}$ and reach up to $50 \%$ for $\mathrm{AC} .^{3}$ This wide variation in the literature may be related to differences in surgical technique, to the absence of a clear definition of recurrence (radiological versus symptomatic) and to falsely low rates advanced by studies with relatively short follow-ups. Indeed, a clinically significant RCC recurrence can occur many years after surgery, ${ }^{1}$ as is illustrated by our Patient 2 (Table 1) who suffered a recurrence 9 years after her original operation. Nonetheless, even when considering the lower range of reported rates, cyst recurrence remains a frequent event. 


\section{Comparative review of reported surgical strategies devised to address recurrence}

While simple drainage of the cyst through a transsphenoidal fenestration in its anterior wall is considered the standard method of treating $\mathrm{RCC}^{14}$ and $\mathrm{AC},{ }^{3}$ their recurrence rates have led some to advocate an aggressive removal of the cyst's walls. ${ }^{7,8,14}$ This more radical approach is however associated with a greater incidence of both CSF leak and postoperative pituitary dysfunction, with diabetes insipidus occurring in nearly half of patients, in contrast to $9 \%$ of those undergoing non-radical resection. ${ }^{17}$ Other groups have therefore continued to support a more conservative stance, considering redo drainage a lesser morbidity in the long-term. ${ }^{5,15,27}$ Interestingly, some reports even challenge the rationale behind aggressive cyst wall resection, having failed to find an association with a more favourable recurrence profile. ${ }^{17,28}$

On a similar note, instilling absolute alcohol into a RCC's lumen, with a view to induce chemical ablation of its secretory cells, has not been shown to confer an advantage but does, on the other hand, expose the patient to grave risks if a communication exists through the arachnoid mater. ${ }^{17,22}$

Alternative approaches have therefore explored means of avoiding aggressive stripping of the cyst's wall while ensuring its long-term drainage. One such method leaves the sellar floor unrepaired to allow drainage of the cyst's contents into the sphenoid sinus, ${ }^{20}$ while another involves creating a communication with the suprasellar cistern, thereby allowing the cyst's contents to drain into the subarachnoid space instead. ${ }^{9-12}$ The latter technique is obviously associated with an increased risk of CSF leak. Moreover, although definitive evidence is lacking, opinion holds that both openings ultimately seal themselves off through scarring, possibly leading to recurrence nonetheless. ${ }^{7,11,14}$ 
Yet other groups resort to cyst lumen obliteration, notably with autologous adipose tissue. ${ }^{27,29,30}$ Filling the pituitary fossa with fat is thought to block it from re-accumulating with fluid in the short term and to induce scarring in the long term that closes off communications with the subarachnoid space presumed to be at the origin of AC. It can however be advanced that fatpacking of the cyst's cavity (- to be distinguished from sphenoid packing) constitutes in many cases a partially blind re-filling of a space that the surgical intention was effectively to decompress, and that its arguable advantages do not warrant its routine use..$^{9,10}$

A recent publication from our group has shown that residual cystic formations are a very frequent finding (45.9\%) on first follow-up MRI performed at 3-6 months after transsphenoidal surgery for RCC. ${ }^{28}$ This same study also identifies the presence of a residual cyst as the only significant risk factor for disease recurrence, superseding surgical approach, extent of resection and histological findings, none of which reached statistical significance. ${ }^{28}$ Moreover, $40 \%$ of patients who underwent cyst resection harboured a residual cyst on their first follow-up MRI, in contrast to $50 \%$ of patients who underwent a fenestration only. These results suggest that although cyst resection comes at a noteworthy risk of postoperative morbidity (as discussed above), it does not protect from the only presumed significant risk factor for cyst recurrence. It is therefore conceivable that an in situ drain, ensuring a longstanding communication between the cyst's lumen and the subarachnoid space, may have a role to play in the long-term management of these diseases.

\section{Cruciform drain: Concept and findings}

The novel concept that we describe here may well address a number of the shortcomings discussed above. Similar to other techniques, it is also an attempt at avoiding the aggressive 
removal of cyst walls. As a result, none of our patients developed a postoperative posterior pituitary insufficiency. Of those who did develop a postoperative ACTH deficiency, it is interesting to note that none harboured an $\mathrm{AC}$, implicative of the inherently greater degree of surgical manipulation involved in the surgery of RCC and pituitary macroadenomas.

In contrast to other techniques that also address the problem of cyst recurrence by establishing a communication with the suprasellar subarachnoid space, here we use a small piece of tubing with a therapeutic rationale similar to that underlying syringo-subarachnoid shunts ${ }^{31}$ (Fig. 5): The in situ drain allows to siphon away the cyst's eventual re-accumulated contents in a more durable way than with an unaided cisternostomy. Other perceived advantages include the possibility of tailoring a smaller posterior fenestration in the cyst wall than that previously advocated, to a size approximating the drain's diameter (Fig. 1D, Fig. 3B); the fact that the indwelling transcisternal drain will prevent the small posterior fenestration from closing off; and the fact that a small cisternostomy leads to less intracisternal disruption and therefore, presumably, to less intracisternal arachnoid scarring.

Although the drain itself may clog up, as can be the case with other forms of shunt, there still remains the possibility for fluid to circulate freely around the drain. Indeed, as illustrated in Figures $1 \mathrm{D}$ and $3 \mathrm{~B}$, the posterior fenestration, although small, does not strangle the drain. Furthermore, the drain's transverse bar anchors it firmly in its trans-cysto-cisternal position, and guards it from unintentionally sliding deeper into the subarachnoid space during packing. The drain is individually fashioned so that only a small portion of it is in the subarachnoid space. And, although the posterior fenestration is small, it remains a keyhole that is large enough to endoscopically inspect the suprasellar space before drain insertion, in order to avoid the theoretical risk of neurovascular injury. Neuronavigation - usually used during redo 
surgeries in any case - can provide further insight into the position of neurovascular structures lying in, and beyond, the suprasellar space.

The cruciform drain technique is primarily intended for recurrent cystic lesions of the sella with serous contents - which typically represent AC and cases of RCC - and where previous simple drainage failed to provide a durable solution as was the case in three of our patients (1 case of a third recurrence of an AC, 1 case of a first recurrence of an AC, and 1 case of a first recurrence of a RCC). Eventual further candidates may be lesions that are intraoperatively felt to be unamenable to resection at a low risk to the pituitary gland, but in which it is also felt that simple drainage has achieved an insufficient decompression. For example, although a very rare situation, we opted to insert a drain in a case of pituitary macroadenoma, on the basis of our intraprocedural finding that its apical, suprasellar cystic portion failed to descend sufficiently, and therefore might have continued to represent a threat to the patient's vision in case the cyst recollected.

On the other hand, we would not advise that a drain be used in cystic craniopharyngiomas due to their association with chemical meningitis. ${ }^{32,33}$ It is however in any case unlikely that such cysts - with their dense, oily contents - be suitable candidates for cruciform drain placement due to the risk of blockage. Worthy of note, RCC can also present with chemical meningitis but this is a very rare occurrence. ${ }^{6,34}$

Our small cohort however experienced a high rate of postoperative CSF leak (Table 1). These initial observations have rapidly prompted us to revise our protocol in favour of higher-grade repair strategies as proposed in the graded approach by Dehdashti et al., ${ }^{35}$ and notably to employ nasoseptal flaps in the future as we otherwise would during extended endonasal 
approaches. Notwithstanding, all our patients demonstrated full recovery when assessed at 3 postoperative months (Table 1). In all patients, MRI confirmed decompression of the anterior optic apparatus, an unchanged position of the implanted cruciform drain and the absence of radiologically discernible complications (Fig. 4).

\section{Limitations}

This work is only at Stage 1 of the IDEAL model (i.e. Idea phase).$^{18}$ The intention of this report is to illustrate the surgical feasibility of the cruciform drain technique and to propose a therapeutic rationale for its use. Seeing that recurrence may take up to ten years to manifest, ${ }^{1}$ this report does not yet allow to draw conclusions on the technique's ultimate efficacy in preventing cyst recurrence in the long-term.

\section{Conclusion}

The use of the cruciform drain in the transsphenoidal management of cystic lesions of the sella is intended as a novel technique to act as a long-term stoma between the cyst's lumen and the suprasellar CSF space with the aim of preventing cyst recurrence. Still at an early phase of innovation, it is meant to address the shortcomings encountered with other reported methods tackling the same problem. At this juncture, its indications concern recurrent cases of $\mathrm{AC}$ or RCC primarily. It is readily available in any neurosurgical theatre, seeing that it is fashioned from a ventricular catheter, and it does not impact on surgical workflow. The observed postoperative CSF leak rate has prompted us to modify our primary repair protocol for future cases and represents a basis for the technique's further development. Its long-term effect on cyst recurrence will need to be studied over several years. 


\section{References}

1. Kinoshita Y, Tominaga A, Usui S, et al. The long-term recurrence of Rathke's cleft cysts as predicted by histology but not by surgical procedure. J Neurosurg. 2016;125(4):1002-1007.

2. Shin JL, Asa SL, Woodhouse LJ, Smyth HS, Ezzat S. Cystic lesions of the pituitary: clinicopathological features distinguishing craniopharyngioma, Rathke's cleft cyst, and arachnoid cyst. J Clin Endocrinol Metab. 1999;84(11):3972-3982.

3. Zada G, Lopes MBS, Mukundan S, Jr., Laws E, Jr. Sellar Region Arachnoid Cysts. In: Zada G, Lopes MBS, Mukundan S, Jr., Laws ER, Jr., eds. Atlas of Sellar and Parasellar Lesions: Clinical, Radiologic, and Pathologic Correlations. Cham, Heidelberg, New York, Dordrecht, London: Springer; 2016:237-243.

4. Zada G, Lopes MBS, Mukundan S, Jr., Laws ER, Jr. Rathke Cleft Cysts. In: Zada G, Lopes MBS, Mukundan S, Jr., Laws ER, Jr., eds. Atlas of Sellar and Parasellar Lesions: Clinical, Radiologic, and Pathologic Correlations. Cham, Heidelberg, New York, Dordrecht, London: Springer; 2016:211-225.

5. Snyder BJ, Naidich TP, Post KD. Cystic Lesions of the Sella. In: Swearingen B, Biller BM, eds. Diagnosis and Management of Pituitary Disorders. Contemporary Endocrinology. Humana Press; 2008:445-466.

6. Zada G. Rathke cleft cysts: a review of clinical and surgical management. Neurosurg Focus. 2011;31(1):E1.

7. Kim JE, Kim JH, Kim OL, et al. Surgical treatment of symptomatic Rathke cleft cysts: clinical features and results with special attention to recurrence. $J$ Neurosurg. 2004;100(1):33-40. 
8. Laws ER, Kanter AS. Rathke cleft cysts. J Neurosurg. 2004;101(4):571-572; discussion 572.

9. Su Y, Ishii Y, Lin CM, Tahara S, Teramoto A, Morita A. Endoscopic transsphenoidal cisternostomy for nonneoplastic sellar cysts. Biomed Res Int. 2015;2015:389474.

10. Oyama K, Fukuhara N, Taguchi M, Takeshita A, Takeuchi Y, Yamada S. Transsphenoidal cyst cisternostomy with a keyhole dural opening for sellar arachnoid cysts: technical note. Neurosurg Rev. 2014;37(2):261-267; discussion 267.

11. Castle-Kirszbaum MD, Uren B, King J, Wang YY, Goldschlager T. Glimpse into Pathophysiology of Sellar Arachnoid Cysts. World Neurosurg. 2018;119:381-383.

12. Dubuisson AS, Stevenaert A, Martin DH, Flandroy PP. Intrasellar arachnoid cysts. Neurosurgery. 2007;61(3):505-513; discussion 513.

13. Kim E. Symptomatic Rathke cleft cyst: clinical features and surgical outcomes. World Neurosurg. 2012;78(5):527-534.

14. Kanter AS, Sansur CA, Jane JAJ, Jr., Laws ERJ, Jr. Rathke's cleft cysts. Front Horm Res. 2006;34:127-157.

15. Benveniste RJ, King WA, Walsh J, Lee JS, Naidich TP, Post KD. Surgery for Rathke cleft cysts: technical considerations and outcomes. J Neurosurg. 2004;101(4):577584.

16. Cavallo LM, Prevedello D, Esposito F, et al. The role of the endoscope in the transsphenoidal management of cystic lesions of the sellar region. Neurosurg Rev. 2008;31(1):55-64; discussion 64.

17. Aho CJ, Liu C, Zelman V, Couldwell WT, Weiss MH. Surgical outcomes in 118 patients with Rathke cleft cysts. J Neurosurg. 2005;102(2):189-193.

18. McCulloch P, Altman DG, Campbell WB, et al. No surgical innovation without evaluation: the IDEAL recommendations. Lancet. 2009;374(9695):1105-1112. 
19. Vandenbroucke JP, von Elm E, Altman DG, et al. Strengthening the Reporting of Observational Studies in Epidemiology (STROBE): explanation and elaboration. Int $J$ Surg. 2014;12(12):1500-1524.

20. el-Mahdy W, Powell M. Transsphenoidal management of 28 symptomatic Rathke's cleft cysts, with special reference to visual and hormonal recovery. Neurosurgery. 1998;42(1):7-16; discussion 16-17.

21. Voelker JL, Campbell RL, Muller J. Clinical, radiographic, and pathological features of symptomatic Rathke's cleft cysts. J Neurosurg. 1991;74(4):535-544.

22. Lillehei KO, Widdel L, Astete CA, Wierman ME, Kleinschmidt-DeMasters BK, Kerr JM. Transsphenoidal resection of 82 Rathke cleft cysts: limited value of alcohol cauterization in reducing recurrence rates. J Neurosurg. 2011;114(2):310-317.

23. Wait SD, Garrett MP, Little AS, Killory BD, White WL. Endocrinopathy, vision, headache, and recurrence after transsphenoidal surgery for Rathke cleft cysts. Neurosurgery. 2010;67(3):837-843; discussion 843.

24. Potts MB, Jahangiri A, Lamborn KR, Blevins LS, Kunwar S, Aghi MK. Suprasellar Rathke cleft cysts: clinical presentation and treatment outcomes. Neurosurgery. 2011;69(5):1058-1068; discussion 1068-1057.

25. Mukherjee JJ, Islam N, Kaltsas G, et al. Clinical, radiological and pathological features of patients with Rathke's cleft cysts: tumors that may recur. J Clin Endocrinol Metab. 1997;82(7):2357-2362.

26. Solari D, Cavallo LM, Somma T, et al. Endoscopic Endonasal Approach in the Management of Rathke's Cleft Cysts. PLoS One. 2015;10(10):e0139609.

27. Baskin DS, Wilson CB. Transsphenoidal treatment of non-neoplastic intrasellar cysts. A report of 38 cases. J Neurosurg. 1984;60(1):8-13. 
28. Marcus HJ, Borg A, Hussein Z, et al. Rathke's cleft cysts following transsphenoidal surgery: long-term outcomes and development of an optimal follow-up strategy. Acta Neurochir (Wien). 2020;162(4):853-861.

29. McLaughlin N, Vandergrift A, Ditzel Filho LF, et al. Endonasal management of sellar arachnoid cysts: simple cyst obliteration technique. J Neurosurg. 2012;116(4):728740.

30. Hasegawa M, Yamashima T, Yamashita J, Kuroda E. Symptomatic intrasellar arachnoid cyst: case report. Surg Neurol. 1991;35(5):355-359.

31. Soleman J, Roth J, Constantini S. Syringo-subarachnoid shunt: how I do it. Acta Neurochir (Wien). 2019;161(2):367-370.

32. Hakizimana D, Poulsgaard L, Fugleholm K. Chemical meningitis from a leaking craniopharyngioma: a case report. Acta Neurochir (Wien). 2018;160(6):1203-1206.

33. Blomstedt GC. Post-operative aseptic meningitis. Acta Neurochir (Wien). 1987;89(34):112-116.

34. Mrelashvili A, Braksick SA, Murphy LL, Morparia NP, Natt N, Kumar N. Chemical meningitis: a rare presentation of Rathke's cleft cyst. J Clin Neurosci. 2014;21(4):692-694.

35. Dehdashti AR, Stofko D, Okun J, Obourn C, Kennedy T. Endoscopic Endonasal Reconstruction of Skull Base: Repair Protocol. J Neurol Surg B Skull Base. 2016;77(3):271-278. 


\section{Table legend}

Table 1. Patient demographic information, diagnoses and postoperative outcome. Abbreviations: $C S F$ cerebrospinal fluid; $L D$ lumbar drain; $K S$ Karnofsky performance score.

\section{Figure legends}

Figure 1. Transsphenoidal endoscopic view through right nostril of Patient 5 from Table 1. The view is directed superiorly towards the suprasellar cystic portion of the tumour that still remains intact and walled off. The cyst's antero-inferior pseudocapsule is pierced with a pituitary knife and its clear serous contents are voided (A-B). This opening into the inferior aspect of the cyst is enlarged using a spatula dissector, allowing to visualise the supero-posterior aspect of the cyst's wall, adherent to arachnoid, in the depth (C). Again using a spatula dissector, a small fenestration (seen in the depth at 3 o'clock) is gently created through the arachnoid into the suprasellar subarachnoid space (D). It is through this fenestration that the tip of the cruciform drain is inserted as illustrated in Figure 2B-C.

Figure 2. The cruciform drain is fashioned from the tip of a ventricular catheter, by making a small vertical incision (along the drain's long axis), through which a second smaller section of drain is inserted. The steps are illustrated using (A) a Silverline ${ }^{\circledR}$ Ventricular Shunt Catheter (Spiegelberg; Hamburg, Germany) seen in the figures that follow. The inset in panel (A) shows the catheter's whole length with its stylet in place. (B-C) The stylet is removed and the catheter's tip is cut to the estimated length required for the cruciform drain. This piece can be brought into the surgical field for an in situ assessment of length; it can be further shortened if 
necessary. The length shown here $(1.8 \mathrm{~cm})$ is the length of the cruciform drain shown in Figures 3 and 5. The in situ assessment of the long-piece also allows to determine the position of the crosspiece and counting the catheter tip's holes can be used to aid in this. (D) A small vertical incision is made through both walls of the drain. Here (as in Figures 3 and 5), it is centred on the drain's $5^{\text {th }}$ hole and extends from its $4^{\text {th }}$ to its $6^{\text {th }}$ holes. (E) A smaller section of drain is cut from the original catheter; it typically measures just under $1 \mathrm{~cm}$ although this too can be tailored to the anatomical needs of the individual patient and their cyst. This piece is then transversally introduced through the incision made in the drain's long-piece. It can either be fitted through manually or with the aid of the original catheter's stylet $(\mathbf{F}-\mathbf{I})$. The inset in panel F shows the cross-piece sheathed over the stylet first, following which the stylet is inserted through the incisions made through the long-piece. The resultant cruciform drain is seen in $(\mathrm{J})$, and the insets show views of the drain from above and the side.

Figure 3. Same view in same patient, as described for Figure 1. The transnasal corridor is wide enough to bring the resulting cross-shaped drain into the surgical field (A). The drain is inserted into the suprasellar subarachnoid space (B-C) through the fenestration made in the cyst illustrated in Figure 1D. The drain, with holes along its entire length, effectively straddles the cyst's lumen and the suprasellar cistern.

Figure 4. Preoperative (left panels) and postoperative MRI (coronal slices) performed at 3-6 months (right panels) for Patients 1 (A), 2 (B), 3 (C), 4 (D) and 5 (E) from Table 1, demonstrating decompression of the anterior optic apparatus.

Figure 5. The trans-cysto-cisternal position of the cruciform drain can be appreciated on (A) a reconstructed oblique coronal CT slice (with an anteroposterior view of the drain) windowed 
for parenchyma, and on (B) a reconstructed oblique CT sagittal slice (with a left lateral view of the drain) windowed for bone. Both CT slices are from the patient in Figures 1 and 2. 
Figure 1.

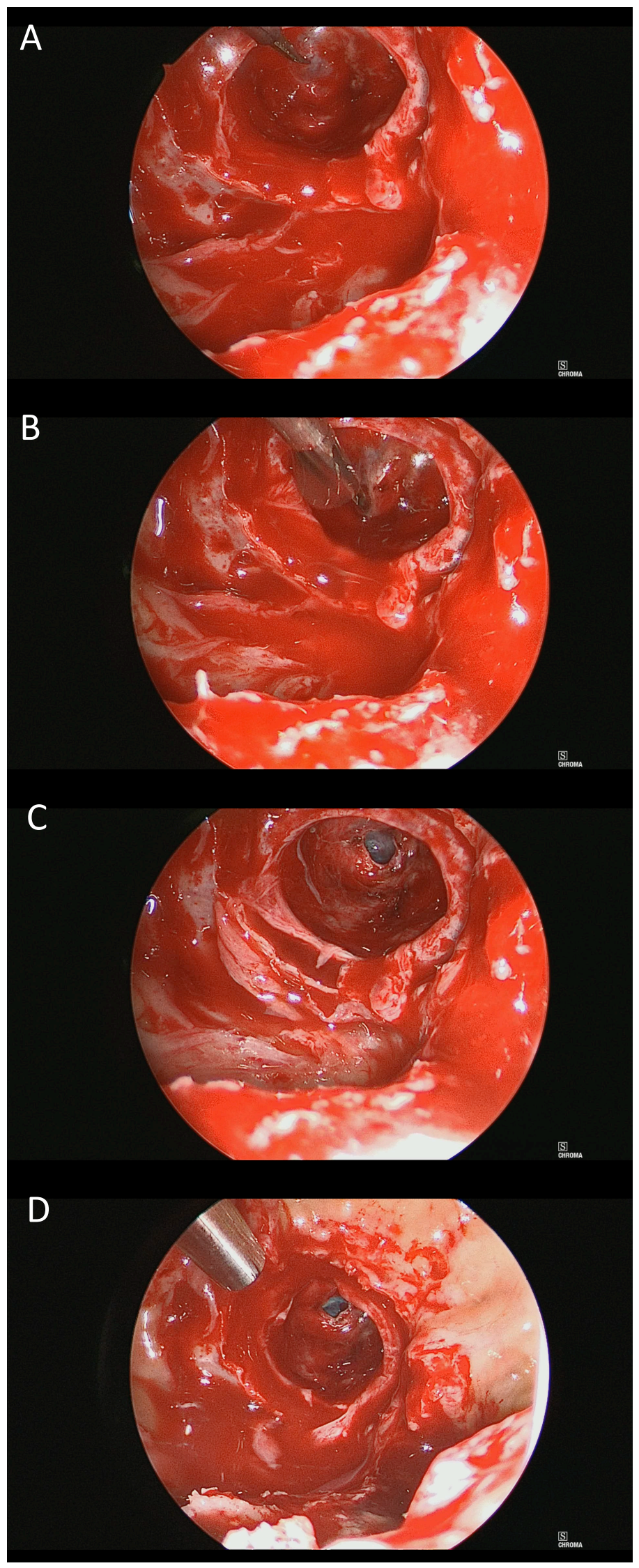


Figure 2.
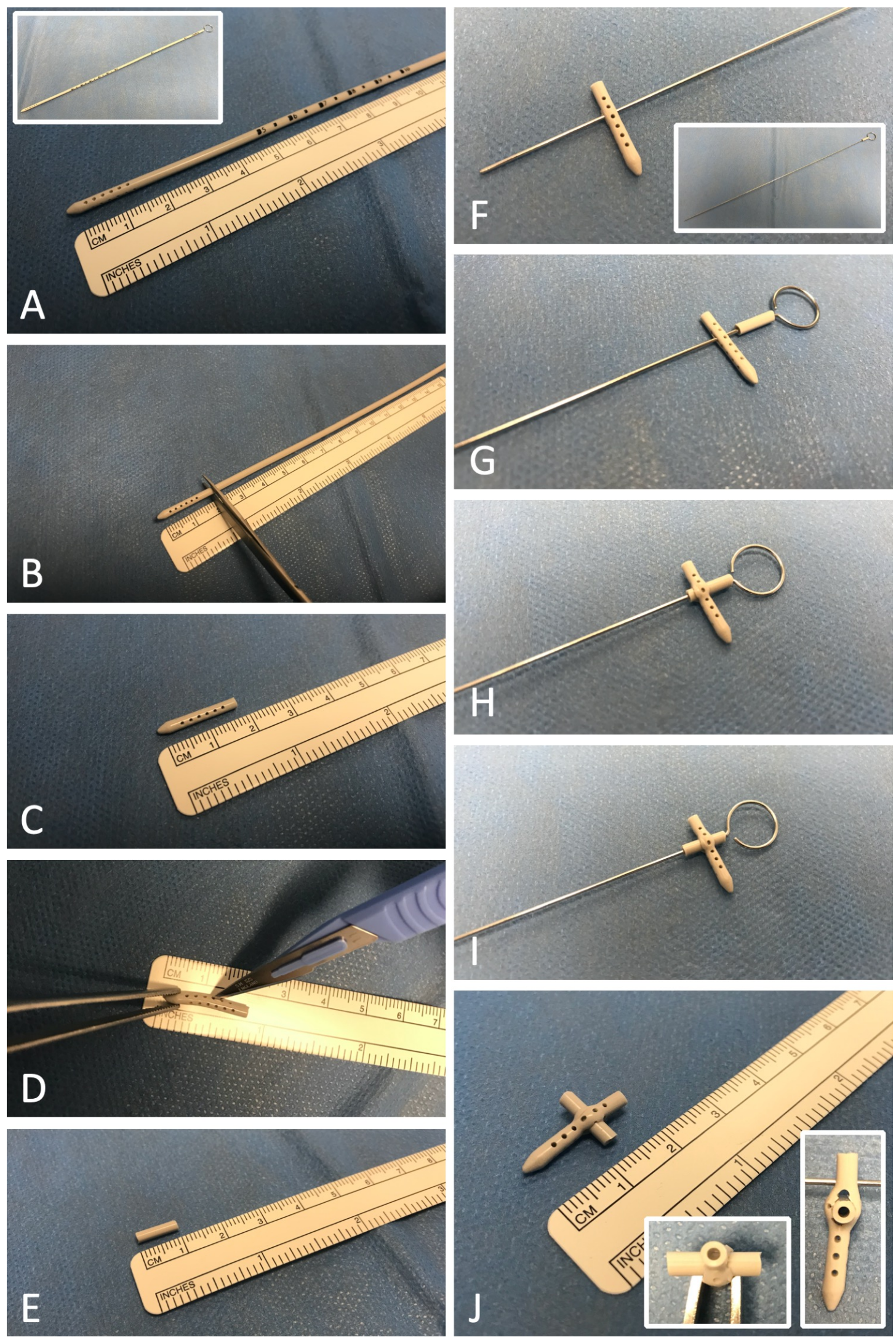
Figure 3.

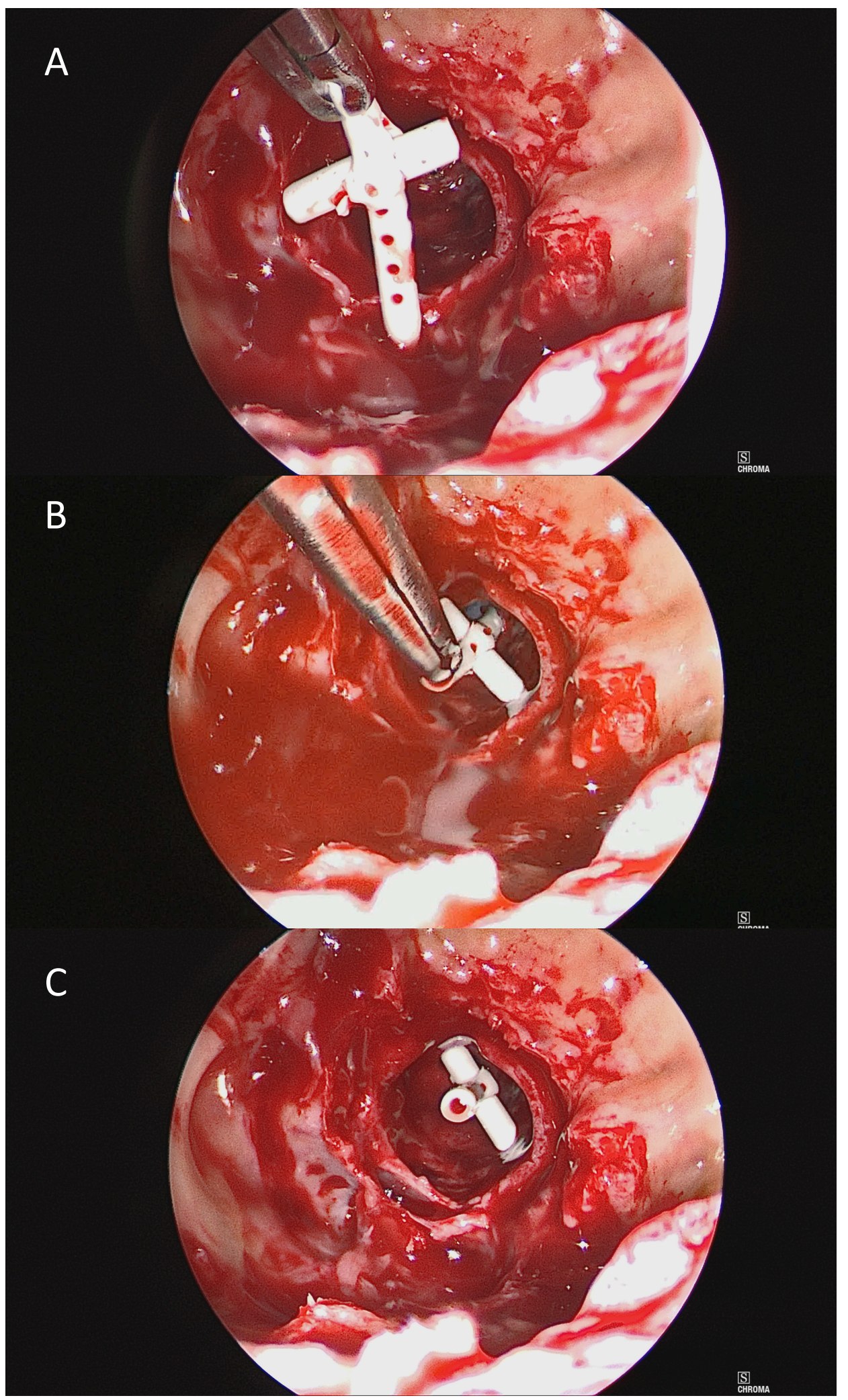


Figure 4.
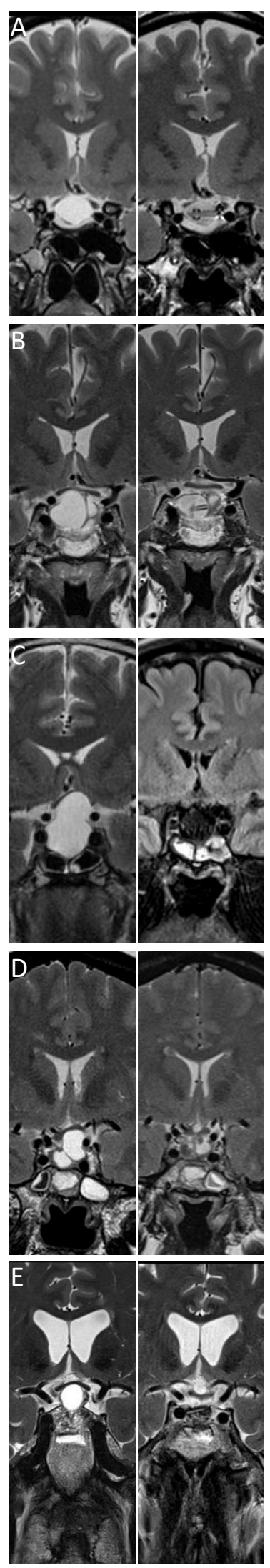
Figure 5.
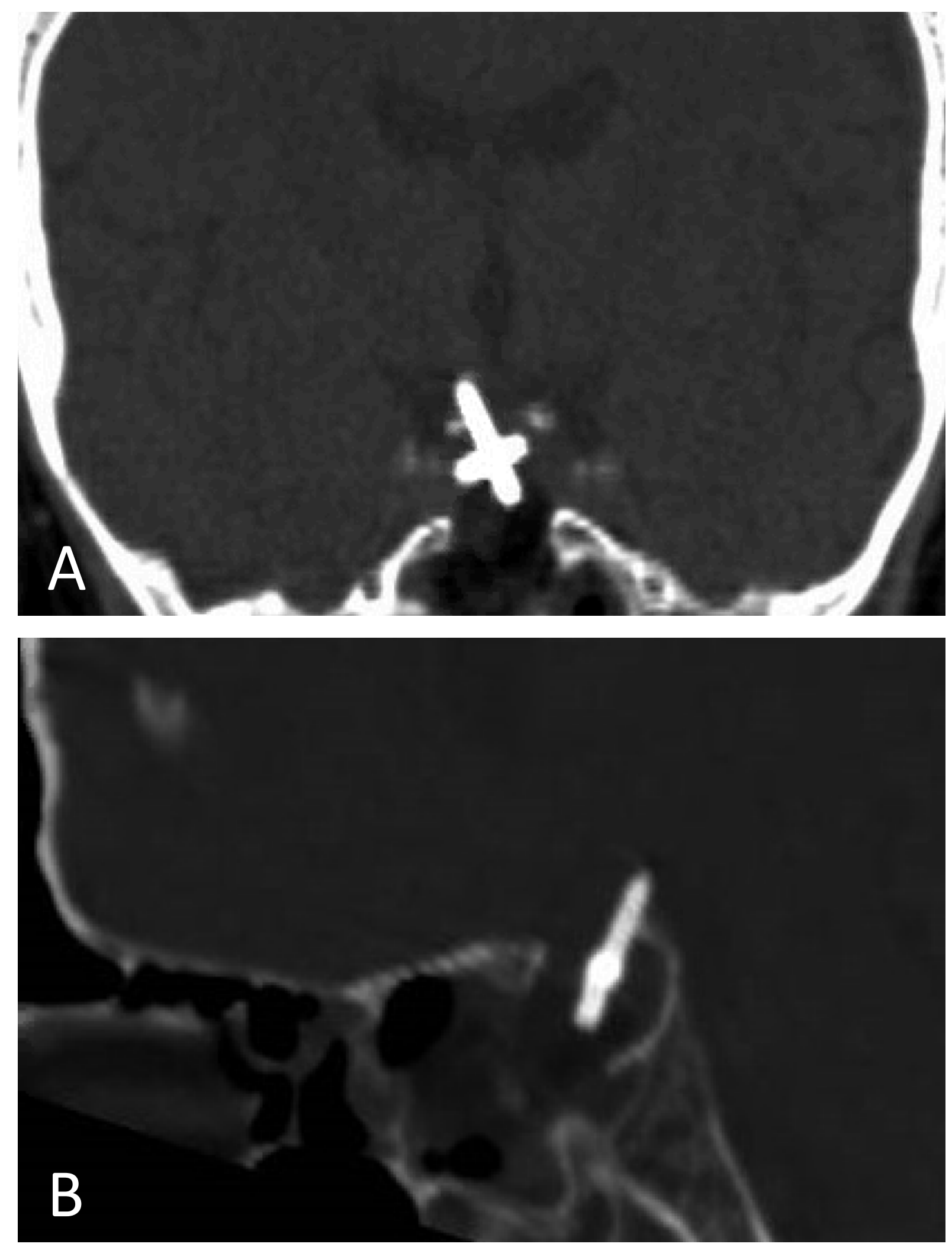
Table 1.

\begin{tabular}{|c|c|c|c|c|c|}
\hline 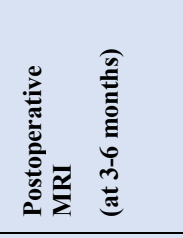 & 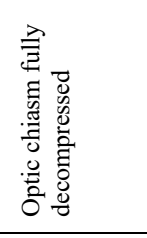 & 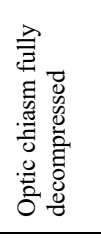 & 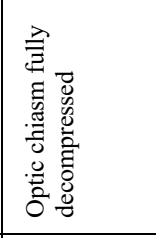 & 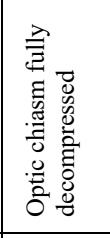 & 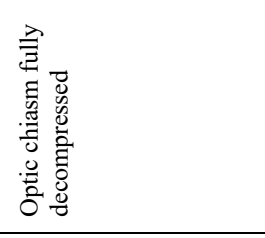 \\
\hline 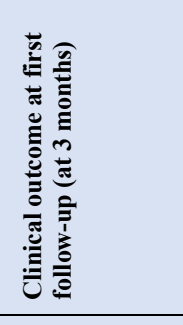 & 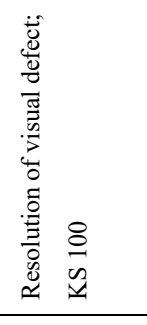 & $\begin{array}{l}\frac{8}{9} \\
\stackrel{y}{1}\end{array}$ & 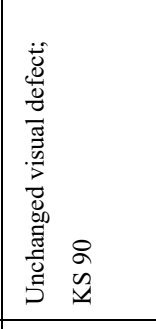 & 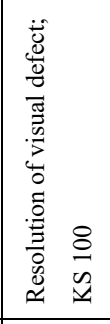 & $\frac{8}{0}$ \\
\hline 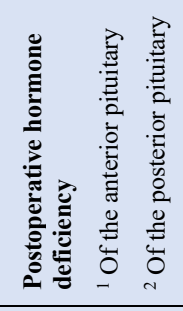 & 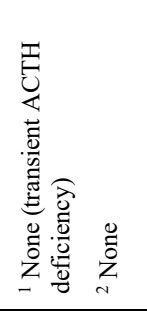 & 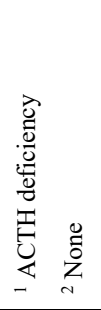 & 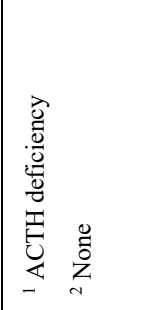 & 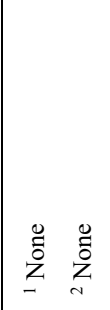 & 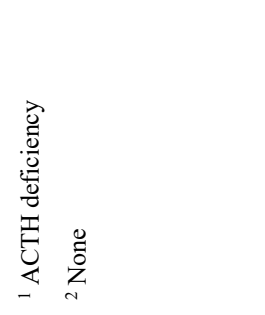 \\
\hline 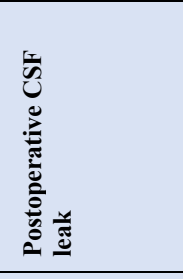 & 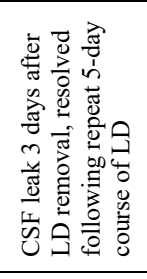 & 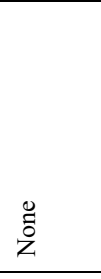 & 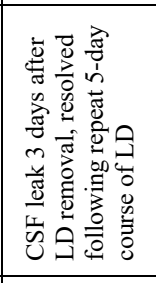 & 总 & 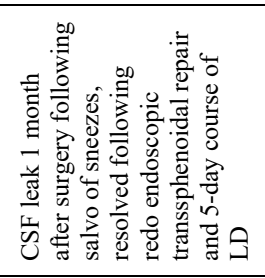 \\
\hline 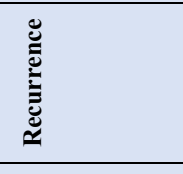 & 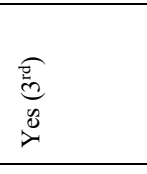 & 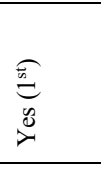 & $\stackrel{\circ}{2}$ & 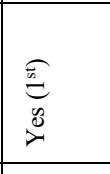 & $\stackrel{\circ}{\check{z}}$ \\
\hline 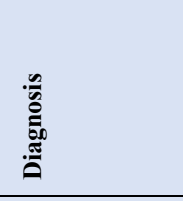 & 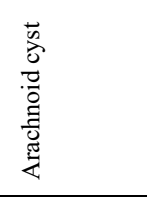 & 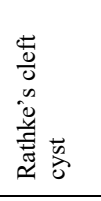 & 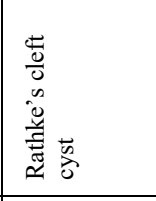 & 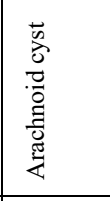 & 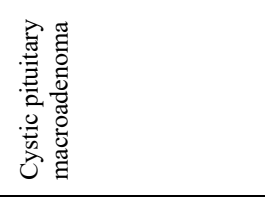 \\
\hline 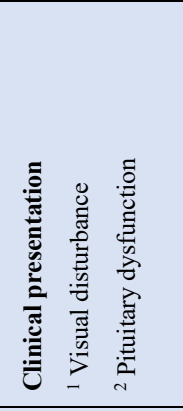 & 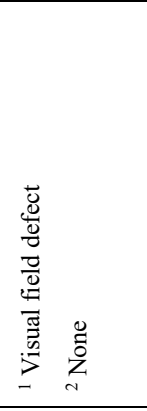 & 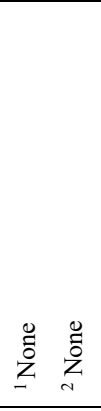 & 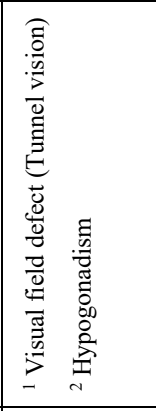 & 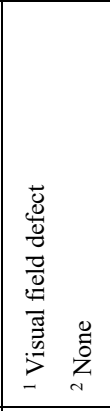 & 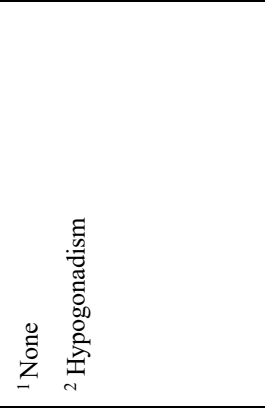 \\
\hline 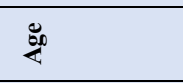 & $\mathcal{F}$ & 8 & g & g & $\vec{n}$ \\
\hline ڤ̆ & i & w & $\Sigma$ & 4 & $\Sigma$ \\
\hline 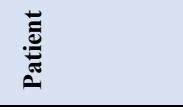 & - & $N$ & $m$ & a & in \\
\hline
\end{tabular}

